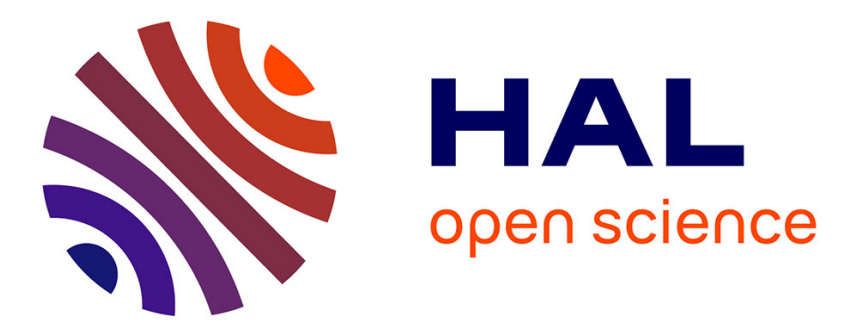

\title{
On the Transport Properties of Li-TFSI Water-in-Salt Electrolytes
}

\author{
Z. Li, R. Bouchal, T. Mendez-Morales, A.-L. Rollet, C. Rizzi, S. Le Vot, F. \\ Favier, B. Rotenberg, O. Borodin, O. Fontaine, et al.
}

\section{- To cite this version:}

Z. Li, R. Bouchal, T. Mendez-Morales, A.-L. Rollet, C. Rizzi, et al.. On the Transport Properties of Li-TFSI Water-in-Salt Electrolytes. Journal of Physical Chemistry B, 2019, 123 (49), pp.10514-10521. 10.1021/acs.jpcb.9b08961 . hal-02412648

\section{HAL Id: hal-02412648 https://hal.science/hal-02412648}

Submitted on 6 Nov 2020

HAL is a multi-disciplinary open access archive for the deposit and dissemination of scientific research documents, whether they are published or not. The documents may come from teaching and research institutions in France or abroad, or from public or private research centers.
L'archive ouverte pluridisciplinaire HAL, est destinée au dépôt et à la diffusion de documents scientifiques de niveau recherche, publiés ou non, émanant des établissements d'enseignement et de recherche français ou étrangers, des laboratoires publics ou privés. 


\title{
On the Transport Properties of Li-TFSI Water-in-Salt Electrolytes
}

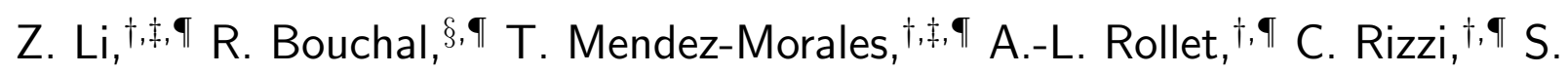
Le Vot,,$\S$ F. Favier,,$\S$ B. Rotenberg, ${ }^{\dagger,}$ O. Borodin, $\|, \perp$ O. Fontaine, ${ }^{\S} \uparrow$ and M.

$$
\text { Salanne }{ }^{*, \dagger,+, \uparrow}
$$

$\dagger$ †orbonne Université, CNRS, Physico-chimie des Électrolytes et Nanosystèmes Interfaciaux, PHENIX, F-75005 Paris, France

$\ddagger$ Maison de la Simulation, CEA, CNRS, Univ. Paris-Sud, UVSQ, Université Paris-Saclay, F-91191 Gif-sur-Yvette, France

【Réseau sur le Stockage Electrochimique de l'Energie (RS2E), FR CNRS 3459, 80039 Amiens Cedex, France

§Institut Charles Gerhardt Montpellier, UMR 5253, CC 1701, Université Montpellier, Place Eugène Bataillon, 34095 Montpellier Cedex 5, France

|| Joint Center for Energy Storage Research, U.S. Army Research Laboratory, Adelphi, MD 20783, USA

$\perp$ Electrochemistry Branch, Sensors and Electron Devices Directorate, U.S. Army Research Laboratory, Adelphi, MD 20783, USA

E-mail: mathieu.salanne@sorbonne-universite.fr

\begin{abstract}
Water-in-salts are a new family of electrolytes that may allow the development of aqueous Li-ion batteries. They have a structure which is reminiscent of the one of ionic
\end{abstract}


liquids, and they are characterized by a large concentration of ionic species. In this work we study their transport properties and how they evolve with concentration by using molecular dynamics simulations. We first focus on the choice of the force field. By comparing the simulated viscosities and self diffusion coefficients with experimental measurements, we select a set of parameters that reproduces well the transport properties. We then use the selected force field to study in detail the variations of the self and collective diffusivities of all the species as well as the transport number of the lithium ion. We show that correlation between ions and water play an important role over the whole concentration range. In the water-in-salt regime, the anions form a percolating network which reduces the cation-anion correlations and leads to rather large values for the transport number compared to other standard electrolytes.

\section{Introduction}

Liquid electrolytes can be classified according to their ionic concentrations. Two main families are generally used in electrochemical devices. In conventional electrolytes, the major species is a neutral molecule and a few, highly solvated ions are present; seawater is an archetypal example. On the contrary, ionic liquids (ILs) are electrolytes that have gained more and more attraction for energy storage applications over the past two decades. ${ }^{1}$ However, they suffer from a rather large viscosity, ${ }^{2}$ which hinders their use at relatively low temperatures (and even at room temperature). Indeed, in the case of electrochemical doublelayer capacitors or in Li-ion batteries a vast majority of the commercial devices employ organic solvent-based electrolytes. ${ }^{1,3,4}$

Recently, a third class of electrolytes was proposed, in which the ionic species are dissolved in large amounts in the solvent: ${ }^{5}$ these are called "solvent-in-salts". This requires to find salts with a good solubility. For example, Suo et al. showed that such concentrated electrolytes

could involve lithium cations by using $\mathrm{Li}\left[\left(\mathrm{CF}_{3} \mathrm{SO}_{2}\right)_{2} \mathrm{~N}\right]$ (LiTFSI). Due to the very low lattice energy of this salt, it can be mixed up to very large amounts with organic solvents. This work 
was extended to a series of other Li salts and solvents, ${ }^{6-8}$ opening the way for fast-charging lithium ion batteries.

Among the potential solvents, water is often discarded in electrochemical energy storage applications because of its narrow electrochemical stability window (ESW) of $1.23 \mathrm{~V}$, which does not allow neither high voltages nor high energies to be reached. This limitation was overcome through the introduction of a LiTFSI-based "water-in-salt" electrolyte, ${ }^{9}$ for which the window could be extended to $\approx 4.0 \mathrm{~V} .{ }^{10}$ Such an important breakthrough may lead to the development of aqueous rechargeable Li-ion batteries. It was shown in this work that this large stability was actually achieved through the formation of an electrode-electrolyte interphase during the first cycles. The mechanisms at the origin of this interphase were then probed in further studies. ${ }^{11}$ More recently, a new concept of aqueous battery was also proposed, which is based on the conversion and intercalation of halogen ions. ${ }^{12}$ This was enabled by another interesting property of water-in-salts, namely their partial immiscibility one with each other provided that the anion have a different shape and size. ${ }^{13}$

In this work, we focus on the physico-chemical properties of a prototypal water-in-salt, using LiTFSI as the conducting salt. Although the transport properties, such as the viscosity, the electrical conductivity or the diffusion coefficients were reported in several works, ${ }^{9,14}$ their variation with concentration and their values in the water-in-salt regime were not put in perspective with respect to existing families of electrolytes. In a first step, we validate the choice of a classical force field that allows to reproduce the experimental values for all these quantities with a good agreement (additional experimental data were gathered for the viscosity, the electrical conductivity and the diffusion coefficients to match the simulation conditions exactly). We then analyze the self and collective diffusion coefficients of waterin-salts using (and partly extending) the methodology introduced by Kashyap et al. ${ }^{15}$ to compare charge transport in ionic liquids and electrolyte solutions. At high salt concentration, despite the presence of a small fraction of "free" water molecules, the system may be considered as an ionic liquid in which the cation is the hydrated lithium ion. We show the 
impact of this structure on the dynamic correlations between the various species, and how it influences the lithium transport number.

\section{Method}

\section{Molecular dynamics simulations}

Table 1: Simulation cell parameters.

\begin{tabular}{cccc}
\hline Molality $\left(\mathrm{mol} \mathrm{kg}^{-1}\right)$ & Cell length $(\AA)$ & $\mathrm{N}($ LiTFSI $)$ & $\mathrm{N}\left(\mathrm{H}_{2} \mathrm{O}\right)$ \\
0.3 & 35.88 & 8 & 1480 \\
3.5 & 28.38 & 32 & 507 \\
7.0 & 31.34 & 64 & 507 \\
12.0 & 29.16 & 64 & 296 \\
15.0 & 35.57 & 128 & 473 \\
20.0 & 34.70 & 128 & 355 \\
\hline
\end{tabular}

The simulation cells consist of mixtures of water molecules and LiTFSI salt, for six different molalities of LiTFSI ranging from 0.3 to $20 \mathrm{~mol} \mathrm{~kg}^{-1}$ (the unit is noted $\mathrm{m}$ in the following). The cells were cubic and constructed using experimental densities; the details are provided in Table 1. The MD simulations were performed using LAMMPS molecular dynamics simulation package. ${ }^{16}$ The SPC/E model was used for water. ${ }^{17}$ Several atomistic force fields $(\mathrm{FF})$ were considered for the TFSI anion, the corresponding parameters are detailed in the next section. The Lennard-Jones parameters we used for Li cation were taken from Reference 18. The simulations were conducted in the NVT ensemble, with the temperature set at $298.15 \mathrm{~K}$. For all of the simulations, the velocity-Verlet algorithm with a time step of $1 \mathrm{fs}$ was used to integrate the equations of motion. The atomic coordinates were saved every 1 ps for post-analysis. Equilibration runs of 30-60 ns were firstly conducted, the dynamics properties were then obtained from the production runs with timescale over 60-110 ns, in which the calculated viscosity, conductivity and diffusion coefficients are well converged (see Supplementary Figures S1 and S2). The simulation lengths for the optimal 
TFSI FF parameters (see below) were extended up to 240 ns at higher concentrations to get better converged collective dynamics.

\section{Density and viscosity measurements}

The density of the solutions $(\rho)$ was measured at 298 and $323 \mathrm{~K}$ with an Anton Paar DSA $5000 \mathrm{M}$ densimeter using the oscillating U-tube method. The cell temperature is controlled by two integrated Pt 100 thermometers together with Peltier elements and an additional reference oscillator to provide long term stability and precise measurements over the selected temperature range. The apparatus was calibrated using distilled water and ambient air.

Viscosities $(\eta)$ were also measured at 298 and $323 \mathrm{~K}$ with an automated AMVn Anton Paar microviscometer. This apparatus was based on the Hoeppler's falling ball principle. A stainless steel ball with a diameter of $1.5 \mathrm{~mm}$ and density $\rho_{\text {ball }}$ rolls down the inside of inclined, sample-filled glass capillary with an internal diameter of either 1.6 or $1.8 \mathrm{~mm}$. The apparatus is equipped with an automatic timer so the time taken by the ball to roll a fixed distance between two magnetic sensors allows to evaluate the viscosity of the fluid mixture. The accuracy and the precision of the measured time are below $0.002 \mathrm{~s}$ and $\pm 0.001 \mathrm{~s}$, respectively. The capillary was placed in a block, at a temperature fixed by a Peltier thermostat with a temperature stability of $\pm 0.01 \mathrm{~K}$. Apparatus calibration was made using the $\mathrm{S} 3$ and N14 certified viscosity standards, which was provided by CANNON instrument company. The falling ball is accelerated by gravity but slowed down by buoyant and friction forces (Stokes'law). Steady velocity is then reached when net force is zero. The viscosimeter constant $K$ defined according to equation 1 had to be determined at each temperature at various angles of inclination, knowing the viscosity $\eta_{\text {standard }}$ and the density $\rho_{\text {standard }}$ of the standard used at this temperature.

$$
K=\frac{\eta_{\text {standard }}}{t\left(\rho_{\text {ball }}-\rho_{\text {standard }}\right)}
$$

where $t$ stands for the rolling ball time. Standard deviation and correlation coefficient were 
calculated on the experimental ball rolling times for each temperature. The error on dynamic viscosity values could be estimated to be below $1 \%$.

\section{Diffusion coefficients measurements}

The diffusion coefficients of the various species were measured using the pulsed field gradient (PFG) NMR technique on the following nuclei: ${ }^{1} \mathrm{H}$ for water, ${ }^{19} \mathrm{~F}$ for TFSI anion and ${ }^{7} \mathrm{Li}$ for lithium cation. The NMR spectra were recorded using two Bruker Avance III NB spectrometers operating at $7.05 \mathrm{~T}$ and $11.7 \mathrm{~T}$ and with a BBFO probe equipped with $50 \mathrm{G} \mathrm{cm}^{-1}$ gradient coil. The lock was obtained with a sealed $2.0 \mathrm{~mm}$ capillary filled with $\mathrm{D}_{2} \mathrm{O}$ inserted in the NMR tube. We used a NMR pulse sequence combining bipolar gradient pulses and stimulated echo. ${ }^{19}$ This sequence is repeated with 16 gradients of increasing

strength $\left(0<g<50 \mathrm{G} \mathrm{cm}^{-1}\right)$, the gradient application time and the diffusion time were adjusted to each nucleus and sample. The self-diffusion coefficients were obtained by nonlinear least-square fitting of the echo attenuation. The temperature regulation of the samples was performed using an air flux at the required temperature.

\section{Electrical conductivity measurements}

The ionic conductivity of the electrolytes was determined by electrochemical impedance spectroscopy measurements using a two-electrode cell with a frequency sweep ranging from $5 \mathrm{MHz}$ to $50 \mathrm{MHz}$ and with a potential amplitude of $10 \mathrm{mV}$. Measurements were performed at $298 \mathrm{~K}$ and the solutions were degassed for $10 \mathrm{~min}$ under nitrogen before all measurements. The ionic resistance is determined as the curve intercepts with the real part of the impedance axis. 


\section{Results and discussion}

\section{Choice of the force field}

We tested four different force fields in order to determine which one better represents the dynamic properties of the liquid. Since our aim is to focus on high concentrations, we chose a molality of $15 \mathrm{~m}$ for this comparison. The parameters of the force fields are either directly taken or slightly derived (by scaling the charges) from the literature:

1. FF1: Intermolecular parameters of TFSI anion are taken from Reference 2 (the charge of Li cation and TFSI anion are +1 and -1 , respectively);

2. FF2: Charges of Li cation and TFSI anion are uniformly scaled by a factor of 0.8 w.r.t. FF1, Lennard-Jones parameters are kept the same;

3. FF3: Intermolecular parameters of TFSI anion are taken from Reference 20 (the charge of Li cation and TFSI anion are +1 and -1 , respectively);

4. FF4: Charges of Li cation and TFSI anion are uniformly scaled by a factor of 0.8 w.r.t. FF3, Lennard-Jones parameters are kept the same.

The corresponding partial charges and Lennard-Jones parameters for the four force fields are listed in Table 2.

The viscosity is calculated from the integral over time of the stress tensor auto-correlation function,

$$
\eta=\beta V \int_{0}^{\infty}\langle\Sigma(0) \Sigma(t)\rangle \mathrm{d} t
$$

where $\beta=1 / k_{B} T$ ( $k_{B}$ being the Boltzmann constant and $T$ the temperature), $V$ the equilibrium volume and $\Sigma$ is an anisotropic element of the stress tensor. An average over the five independent components of the latter is made to improve the statistics. The diffusion 
Table 2: Nonbonded parameters for TFSI anion, where $q$ is the atomic partial charge and $\sum q$ is the total charge that the TFSI anion carries. $\epsilon$ and $\sigma$ are Lennard-Jones parameters in units of $\mathrm{kcal} \mathrm{mol}^{-1}$ and $\AA$, respectively. For FF2 and FF4, the Li cation charges were also rescaled by a factor of 0.8 .

\begin{tabular}{cccccccc}
\hline & & $\mathrm{C}$ & $\mathrm{F}$ & $\mathrm{O}$ & $\mathrm{S}$ & $\mathrm{N}$ & $\sum q$ \\
\hline \multirow{2}{*}{ FF1 } & $q$ & 0.2988 & -0.1176 & -0.3588 & 0.4845 & -0.4258 & -1 \\
& $\epsilon$ & 0.049 & 0.053 & 0.091 & 0.193 & 0.153 & \\
& $\sigma$ & 3.566 & 2.91 & 3.082 & 3.42 & 3.066 & \\
FF2 & $q$ & 0.23904 & -0.09408 & -0.28704 & 0.3876 & -0.34064 & -0.8 \\
& $\epsilon$ & 0.049 & 0.053 & 0.091 & 0.193 & 0.153 & \\
& $\sigma$ & 3.566 & 2.91 & 3.082 & 3.42 & 3.066 & \\
FF3 & $q$ & 0.35 & -0.16 & -0.53 & 1.02 & -0.66 & -1 \\
& $\epsilon$ & 0.066 & 0.053 & 0.21 & 0.25 & 0.17 & \\
& $\sigma$ & 3.50 & 2.95 & 2.96 & 3.55 & 3.25 & \\
FF4 & $q$ & 0.28 & -0.128 & -0.424 & 0.816 & -0.528 & -0.8 \\
& $\epsilon$ & 0.066 & 0.053 & 0.21 & 0.25 & 0.17 & \\
& $\sigma$ & 3.50 & 2.95 & 2.96 & 3.55 & 3.25 & \\
\hline
\end{tabular}

coefficient of any species $A$ is determined using

$$
D_{A}^{s, \mathrm{PBC}}=\lim _{t \rightarrow \infty} \frac{1}{6 t}\left\langle\left|\delta \mathbf{r}_{i}(t)\right|^{2}\right\rangle
$$

where $\delta \mathbf{r}_{i}$ is the displacement of a given atom $i$ of species type $A$ in time $t$ (in the following we note $A=+$ for Li cations, - for TFSI anions and $w$ for water molecules). This expression yields a value which depends on the box size, hence it is noted with the superscript PBC (which stands for periodic boundary conditions). We then obtain the self-diffusion coefficient $D_{A}^{s}$ for an infinite box size using the appropriate correction. ${ }^{21}$ All these quantities are compared with the experimental values on Figure 1. Focusing first on FF1 and FF3, we see that the former yields a much better agreement with experiments concerning the viscosity. This is not surprising since the original FF by Canongia Lopes and Pádua ${ }^{20}$ notoriously yields too viscous dynamics. ${ }^{22,23}$ This is also reflected in the diffusion coefficients of the ions, which are underestimated in both cases, but to a much lesser extent for FF1. However, this force field does not predict accurately the ratio of the diffusivities between the water 

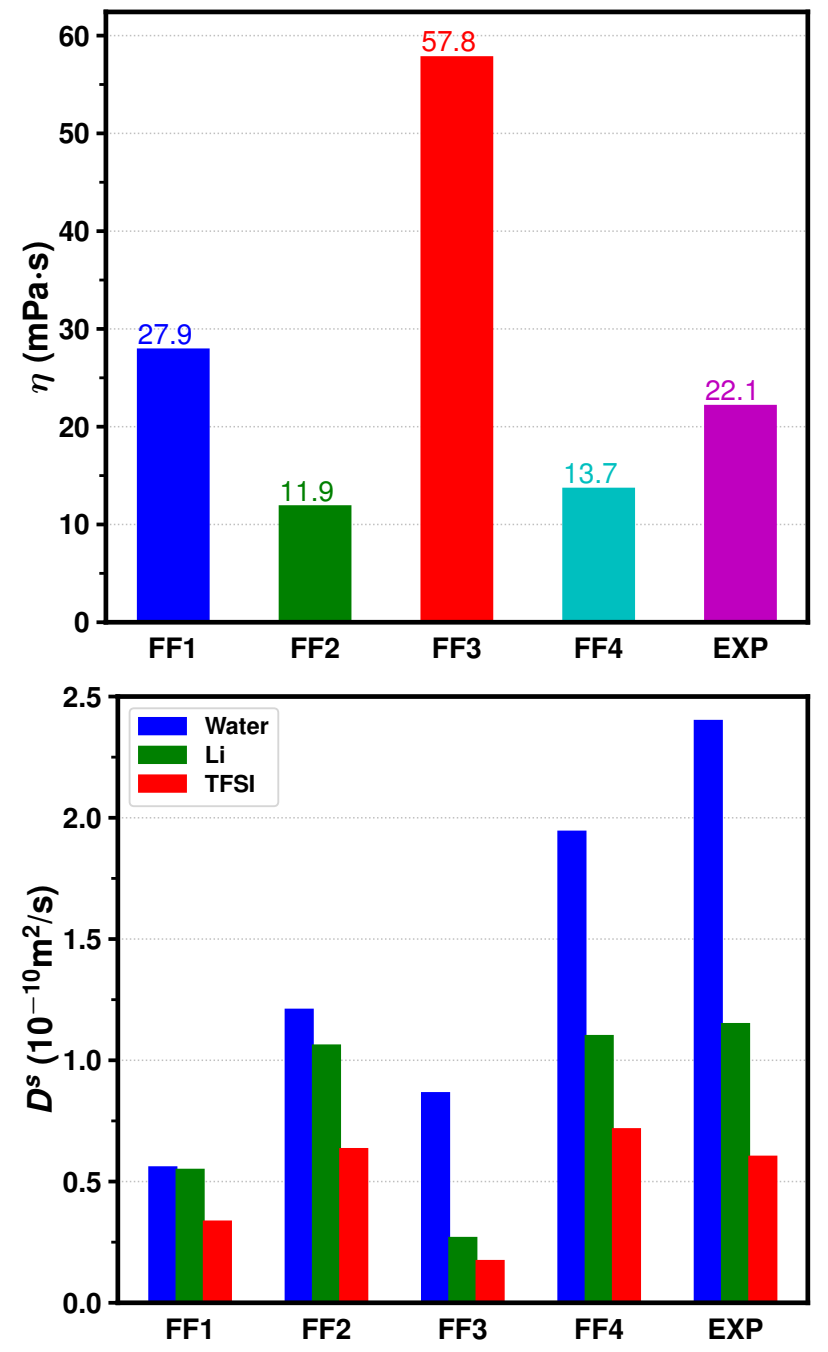

Figure 1: Comparison of the viscosities (top) and the self-diffusion coefficients of Li, TFSI and water (bottom) yielded by the 4 different force fields and the corresponding experimental

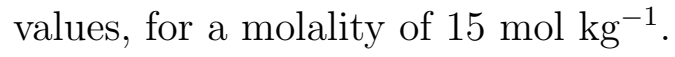

molecules and ionic species. Indeed, it yields a diffusion coefficient for water which is almost equal to the one of the lithium ions, while in experiments there is a factor two between them. FF3 does not show such a behavior and is therefore more accurate for the ratio. This discrepancy can be explained by analyzing the structure of the liquid, and more precisely the solvation shell of the lithium ions. Indeed, as shown in previous studies, ${ }^{9,14}$ most of the water molecules are involved in the solvation shell of a cation at large ionic concentrations, leaving only a few of them uncoordinated. In our case, FF1 predicts an even stronger effect, 
and the concentration of uncoordinated water molecules is almost zero. We can therefore conclude that despite yielding almost correct viscosity and diffusion coefficients of the ions, this force field does not predict correctly the structure of the liquid and the diffusivity of water.

Following previous works, the method consisting in rescaling the ionic charges allows to mimic polarization effects in an effective way. ${ }^{23-28}$ As expected, this yields faster dynamics for both FF2 and FF4, i.e. a decrease of the viscosity and an increase of the diffusion coefficients by factors ranging between 2 and 4 . In both cases, the viscosity is now underestimated w.r.t. the experimental value, but the agreement is rather good. The improvement is much more pronounced for the diffusion coefficients. The lithium and TFSI ones match almost quantitatively with experiments, but again a marked difference is observed for the water diffusivity. FF2 does not improve much the situation observed for FF1, since the value obtained for the water diffusion coefficient is similar to the one of the lithium cation. In contrast, in the case of FF4 we obtain a value which is only $20 \%$ lower than the one extracted from the PFG-NMR experiments.

Based on these extensive comparisons, we have decided to perform the study of the transport properties across the whole range of compositions using FF4. In a first step, we validate this choice by systematically comparing the viscosity, the ionic conductivity and the diffusion coefficients to the experimental values of Suo et al. ${ }^{9}$ and of our own measurements. The conductivity is computed using the following expression:

$$
\sigma=\frac{\beta e^{2}}{V} \lim _{t \rightarrow \infty} \frac{1}{6 t}\left\langle\left|\sum_{i} Z_{i} \delta \mathbf{r}_{i}(t)\right|^{2}\right\rangle
$$

where $Z_{i}$ is the formal charge of ion $i$. As shown on Figure 2, the agreement is almost quantitative for all the concentrations, ranging from rather dilute $\left(0.3 \mathrm{~mol} \mathrm{\textrm {kg } ^ { - 1 }}\right)$ to the

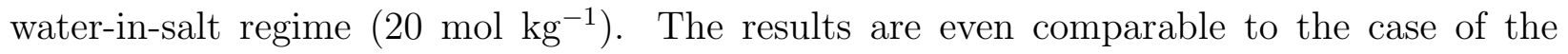
polarizable force field developed by Borodin et al., ${ }^{14}$ as shown in Supplementary Figure S3. 

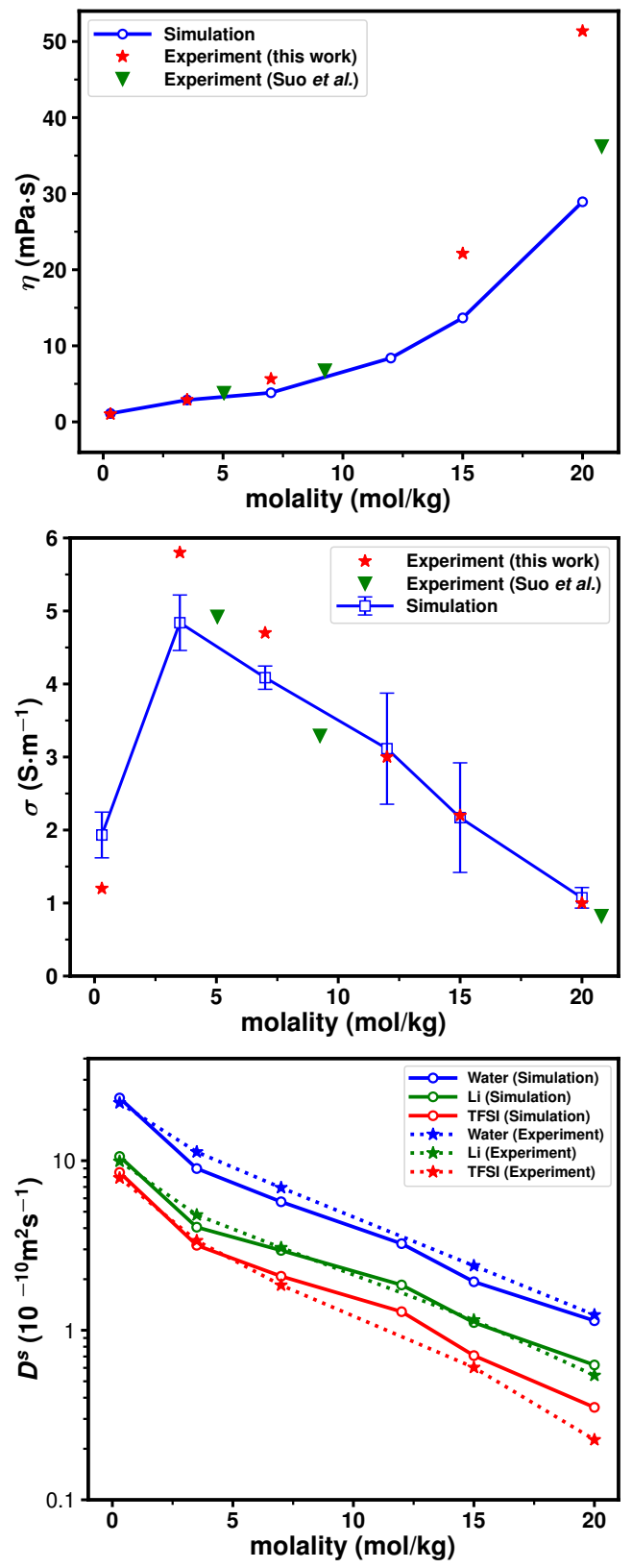

Figure 2: Validation of the FF4 force field across the whole range of composition. The simulated values for the viscosity (top), electrical conductivity (middle) and self-diffusion coefficients (bottom) are compared with the experimental values of Suo et al. ${ }^{9}$ and of the present study.

We can therefore conclude that this force field provides a very accurate representation of the physical properties of the liquid. Note that another parameterization was recently proposed for the TFSI anion, which could also be interesting to test in future works. ${ }^{29}$ 


\section{Ion-ion correlation effects}
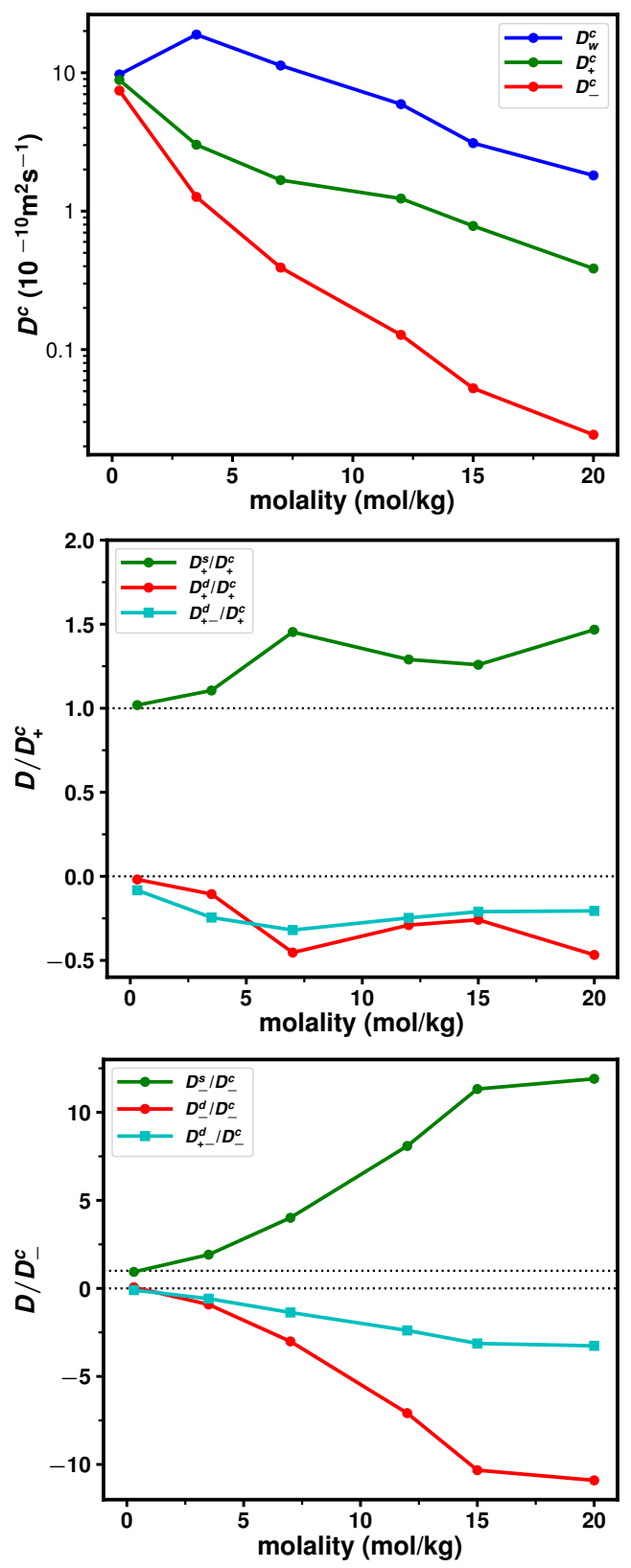

Figure 3: Top: Collective diffusion coefficients for all the species. Middle and bottom: Variation of the self and distinct contributions of the diffusion coefficients for each species, respectively normalized by the collective diffusion coefficient of the cation and the anion.

The measured and calculated physical properties agree with the picture of the highly concentrated systems behaving as an ionic liquid: the electrical conductivities and viscosities (Figure 2) are very similar to the ones of 1-ethyl-3-methylimidazolium TFSI at room 
temperature $^{30}\left(0.83 \mathrm{~S} \mathrm{~m}^{-1}\right.$ and $\left.28 \mathrm{mPa} \mathrm{s}\right)$. In the following we analyze how the peculiar structure of this system affects the dynamic properties and whether these properties are similar to the case of conventional aqueous electrolytes or of ILs.

We focus on the collective diffusion coefficients, which are defined as:

$$
D_{A}^{c}=\frac{1}{N_{A}} \lim _{t \rightarrow \infty} \frac{1}{6 t}\left\langle\left|\sum_{i}^{N_{A}} \delta \mathbf{r}_{i}(t)\right|^{2}\right\rangle
$$

where $N_{A}$ is the number of atoms of species $A$. This quantity differs from the self-diffusion coefficient defined above because it accounts for the motion of all the atoms of a given type. $D_{A}^{c}$ and $D_{A}^{s}$ are equal when the particles move in an uncorrelated way, and any deviation originates from the structural organization of the solution. The difference between the two terms is called the distinct diffusion coefficient $D_{A}^{d}=D_{A}^{c}-D_{A}^{s}$, it only contains these correlations effects. Note that it is also possible to define a distinct cross-term between two species $A$ and $B$ :

$$
D_{A B}^{d}=\frac{1}{\left(N_{A} N_{B}\right)^{1 / 2}} \lim _{t \rightarrow \infty} \frac{1}{6 t}\left\langle\sum_{i}^{N_{A}} \delta \mathbf{r}_{i}(t) \sum_{j}^{N_{B}} \delta \mathbf{r}_{j}(t)\right\rangle
$$

In particular $D_{+-}^{d}$ measures the cation-anion contribution to the electrical conductivity.

We first focus on the variation of the collective diffusion coefficients with respect to the concentration of LiTFSI, which is shown on the top panel of Figure 3. We observe that for water molecules and lithium cations, the absolute values are very similar to the ones of the self-diffusion coefficients across the whole composition range. On the contrary, the TFSI anion collective diffusion coefficient drops very substantially, reaching much lower values in the water-in-salt regime. This shows that the contribution of anions to the overall electrical conductivity is almost negligible, and points towards a very different behavior between the two ionic species.

The various self and distinct contributions to the collective diffusion coefficient may respectively be appreciated on the middle and bottom panels of Figure 3 for the cation and the anion. $D_{+}^{d}$ and $D_{-}^{d}$ are both negative (red lines in Figure 3), which indicates an 
anti-correlated motion of ions of like charge. The ratio with respect to the total/collective one is gradually decreasing with the concentration, starting from a value close to 0 in both cases. It reaches a large absolute value of 10 for the anion, while for the cation it decreases at the beginning and then remains almost constant with a small value (around -0.3) above

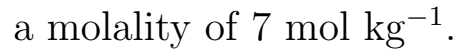

Distinct diffusion coefficient for cation-anion correlation (cyan lines in Figure 3) are negative (anti-correlated) in the whole concentration range, which is in contrast to the results of an aqueous solution reported by Kashyap et al., ${ }^{15}$ who found positive correlations of cationanion for $4.6 \mathrm{~mol} \mathrm{~L}^{-1} \mathrm{NaCl}$. On the contrary, our results are consistent with those reported in the same study for a pure IL and for high temperature molten salts. ${ }^{15}$ In other works focusing on mixtures of ILs with solvents, i.e. water-[BMIM] $\left[\mathrm{BF}_{4}\right]$ mixtures $^{31}$ and solvate ionic liquids $([\mathrm{Li}(\mathrm{G} 4)][\mathrm{TFSI}]),{ }^{32}$ negatively correlated motions of ions of opposite charges were also found at high salt concentrations. Note that the ratio of negatively correlated motion of cationanion with respect to the anion is gradually decreasing with the concentration while it does not change significantly for large concentrations when normalized by $D_{+}^{c}$ instead.

\section{Ion-water correlation effects}

Similarly, it is possible to study the correlations between the motion of the ions and that of the water molecules using the distinct diffusion coefficients, as shown on Figure 4. This quantity displays positive values for cations (i.e. water molecules move together with cations) and the ratio significantly increases with concentration up to $7 \mathrm{~mol} \mathrm{~kg}^{-1}$, then stays almost constant around a value of 1.6-1.7. Such a strong correlation between the motion of lithium cations and water molecules is not surprising since the solvation shell of these ions is very tightly bound in the highly concentrated regime.

On the contrary, we obtain a negative ratio for the water-anion distinct diffusion coefficient, that gradually decreases with the concentration. Large values are observed, which can be assigned to the low values taken by $D_{-}^{c}$ at large LiTFSI concentrations. Nevertheless, the 


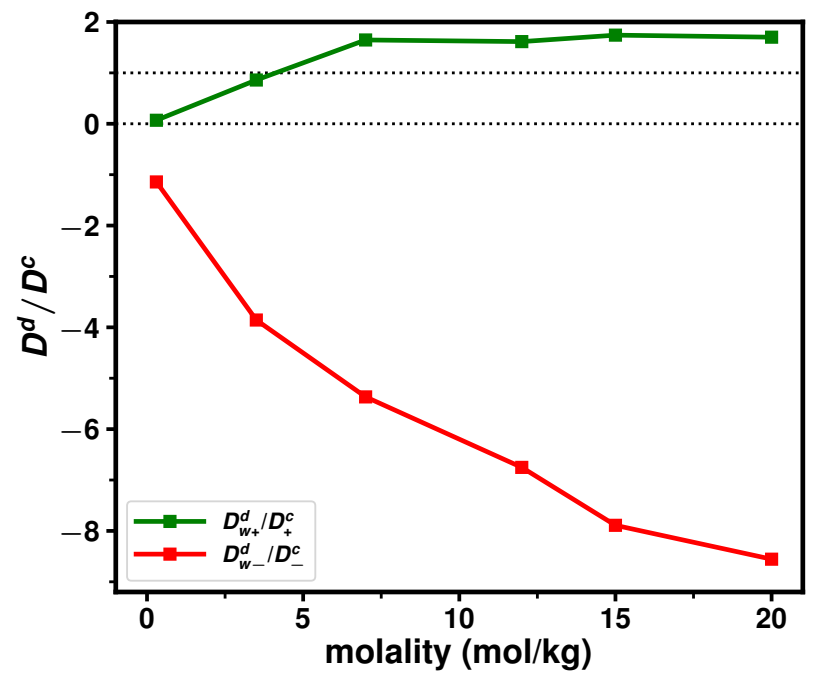

Figure 4: Variation of the water-ion distinct diffusion coefficients. The value is normalized by the corresponding ion collective diffusion coefficient to facilitate comparisons.

anti-correlated motion is again well explained by the structure of the melt, since the ion-rich nanodomains made are primarily comprised of the TFSI-rich salt aggregates, ${ }^{14}$ that only loosely bind water molecules. We can therefore think of the water-in-salt as a liquid where the structure is dominated by an anionic network with a slow relaxation time, and hence a low collective diffusivity, while the water and cations are forming some channels in which they diffuse in a concerted (and relatively fast) way.

\section{Lithium ion transport numbers}

We now analyze how the various correlations impact the collective transport properties, mostly focusing on the transport of lithium ions since they are the main species involved in the functioning of the batteries. To this end, many studies report an apparent transport number, which is defined as

$$
t_{+}^{\text {apparent }}=\frac{D_{+}^{s}}{D_{+}^{s}+D_{-}^{s}}
$$

The qualificative apparent is due to the fact that this quantity only relies on the self-diffusion coefficient, thus neglecting the correlation effects discussed above. Nevertheless it is widely 
Table 3: Transport properties: $\alpha$ and $\beta$ are coefficients that respectively account for the relative collective mobility of the ions and the strength of the cation-anion correlations (Equations 9 and 10); $t_{+}^{\text {abc }}$ is the transport number under anion-blocking conditions (Equation $8) ; t_{+}^{\text {apparent }}$ is the apparent transport number calculated by neglecting all the correlation effects (Equation 7).

\begin{tabular}{lllll}
\hline $\begin{array}{l}\text { Molality } \alpha \\
\left(\mathrm{mol} \mathrm{kg}^{-1}\right)(-)\end{array}$ & $\begin{array}{l}\beta \\
(-)\end{array}$ & $\begin{array}{l}t_{+}^{\text {abc }} \\
(-)\end{array}$ & $\begin{array}{l}t_{+}^{\text {apparent }} \\
(-)\end{array}$ \\
\hline 0.3 & 0.54 & -0.09 & 0.495 & 0.55 \\
3.5 & 0.70 & -0.35 & 0.449 & 0.56 \\
7.0 & 0.81 & -0.52 & 0.3 & 0.59 \\
12.0 & 0.91 & -0.45 & 0.256 & 0.59 \\
15.0 & 0.94 & -0.39 & 0.23 & 0.61 \\
20.0 & 0.94 & -0.39 & 0.223 & 0.64 \\
\hline
\end{tabular}

used because self-diffusion coefficients are now routinely measured in electrolytes using PFGNMR. The values extracted from our simulations are provided in Table $3 . t_{+}^{\text {apparent }}$ is larger than 0.5 on the whole composition range, which is due to the largest diffusivity of $\mathrm{Li}^{+}$ ions compared to the TFSI ones. This number also increases with the salt concentration, which should lead to an increasing efficiency of the lithium transport for Li-ion battery applications. However recent works ${ }^{32-35}$ have shown that this quantity may not predict well the performance of an electrolyte for Li-ion batteries applications.

Indeed, the large extent of correlation effects, not only between cations and anions but also between ions of the same charge, which was evidenced in the previous sections, shows that some caution should be taken when discussing the apparent transport number of lithium. Recently, Wohde et al. combined the Onsager reciprocal relations with linear response theory in order to derive expressions for another transport number, which takes into account all correlations between ionic movements in the electrolyte. ${ }^{33}$ It is noted $t_{+}^{\text {abc }}$, where the superscript abc stands for anion-blocking conditions, which corresponds to the experimental setup for measuring efficiently single cation transport numbers. The definition in terms of collective diffusion coefficients (for an electrolyte made of monovalent ions, $\left|Z_{-}\right|=Z_{+}=1$ ) is

$$
t_{+}^{\mathrm{abc}}=\frac{D_{-}^{c} D_{+}^{c}-\left(D_{+-}^{d}\right)^{2}}{D_{-}^{c}\left(D_{+}^{c}+D_{-}^{c}-2 D_{+-}^{d}\right)}
$$


It may be written by introducing two coefficients that account for the relative collective mobility of the ions and the strength of the cation-anion correlations, which are respectively noted $\alpha$ and $\beta$ and are equal to:

$$
\begin{aligned}
& \alpha=\frac{D_{+}^{c}}{D_{+}^{c}+D_{-}^{c}} \\
& \beta=\frac{2 D_{+-}^{d}}{D_{+}^{c}+D_{-}^{c}}
\end{aligned}
$$

Then the corresponding transport number reads:

$$
t_{+}^{\mathrm{abc}}=\frac{\beta^{2}-4 \alpha+4 \alpha^{2}}{4(1-\alpha)(\beta-1)}
$$

It is worth noting that in the absence of correlations, $D_{+}^{c}=D_{+}^{s}, D_{-}^{c}=D_{-}^{s}$ and $D_{+-}^{d}=0$, leading to $t_{+}^{\mathrm{abc}}=\alpha=t_{+}^{\text {apparent }}$. The various values extracted from our simulations for $t_{+}^{\mathrm{abc}}$, $\alpha$ and $\beta$ are provided in Table 3. We immediately see that $\alpha$ increases markedly with the concentration, reaching values above 0.9 , which reflects the much larger collective diffusion coefficient recorded for the lithium ions. The $\beta$ coefficient is very low at small salt concentration, which is expected since the corresponding system is the closest one to ideality. Due to the anti-correlated motion of cation and anions, it takes negative values at low concentration, which shows that a mechanism of diffusion of the ionic species via the formation of ion pairs can be ruled out. It has a maximal absolute value (i.e. $\beta=-0.52$ ) at intermediate concentration $\left(7 \mathrm{~mol} \mathrm{~kg}^{-1}\right)$ before decreasing in the highly concentrated regime. This decrease, which is significant, is probably due to the formation of the percolating network of anions at these concentrations: Cations and anions are then diffusing separately since they are not well mixed anymore at the nanometer scale. There remains some interaction because the two networks are in contact of each other, but their extent is reduced with respect to a conventional concentrated electrolyte. Note that this structural feature is different from the case of pure ionic liquids. In the latter nanodomains are also formed, but they involve the 
nonpolar and the polar parts of the molecular ions. ${ }^{36-38}$

As a consequence of these complex correlations, the transport number $t_{+}^{\text {abc }}$ takes much lower values than the apparent one, since it is always lower than 0.5. It is largest in the dilute regime and progressively decreases, reaching a value of 0.22 in the most concentrated salt. Although this value means that the transport of lithium is not as efficient as one would have expected based on the diffusivities only, we note that it is relatively large compared to other reported Li-based electrolytes. For example, a value of 0.025 was reported for LiTFSI dissolved in a tetraglyme (G4) solvent. ${ }^{32}$ As discussed above, the relatively small value of $\beta$ for water-in-salts, which is due to the anionic network formation, is one of the key factors that should enable these electrolytes to display good performance in batteries. This can be attributed to the much smaller mass (by a factor of 3.2) of the $\mathrm{Li}\left(\mathrm{H}_{2} \mathrm{O}\right)_{4}^{+}$entity compared to the $\mathrm{Li}^{+}{ }_{-} \mathrm{G} 4$ solvate, which leads to smaller anticorrelations effects with the TFSI anions, as required by the conservation of momentum in the regime in which there is essentially no free solvent available.

\section{Conclusion}

Due to their peculiar structure, the water-in-salts may be seen as intermediate systems between conventional solvent-based electrolytes and pure ionic liquids. Here we have shown that their transport properties (self-diffusion coefficients, conductivities, viscosities) are very well reproduced using classical molecular dynamics simulations with a simple, non-polarizable force field. Such simulations also yield additional information that are not available easily from experiments, such as the collective diffusivities and their decomposition in various self and distinct terms. By analyzing these quantities, we have shown that from the dynamic point of view, the LiTFSI-based water-in-salts are very similar to ionic liquids (pure or mixed with small amounts of solvents). This is not surprising since these systems can be accurately described as mainly consisting in hydrated lithium $\mathrm{Li}\left(\mathrm{H}_{2} \mathrm{O}\right)_{n}^{+}$cations and TFSI anions. Nev- 
ertheless, the spatial organization of the anions, i.e. the formation of nanodomains, strongly impacts transport since the cation-anion dynamic correlations are maximal at intermediate salt concentrations. As a consequence, the lithium transport number is larger than the values previously reported in other representative electrolytes, which reinforces the potential of water-in-salt to be used in future battery applications.

\section{Supporting Information Available}

Running integral of the stress-tensor time correlation function at various compositions for FF4, mean square displacements for all the individual and collective processes at $20 \mathrm{~mol} \mathrm{~kg}^{-1}$ for FF4, comparison of the predicted transport properties by FF4 and a polarizable force field.

\section{Acknowledgements}

This work was supported by the French National Research Agency (Labex STORE-EX, Grant No. ANR-10-LABX-0076). This project has received funding from the European Research Council (ERC) under the European Union's Horizon 2020 research and innovation programme (grant agreement No. 771294). This work was granted access to the HPC resources of CINES under the allocation 2018-A0040910463 made by GENCI. Work at ARL by O. Borodin was supported as part of the Joint Center for Energy Storage Research, an Energy Innovation Hub funded by the U.S. Department of Energy, Office of Science, Basic Energy Sciences through IAA SN2020957.

\section{References}

(1) MacFarlane, D. R.; Forsyth, M.; Howlett, P. C.; Kar, M.; Passerini, S.; Pringle, J. M.; Ohno, H.; Watanabe, M.; Yan, F.; Zheng, W. J.; Zhang, S.; Zhang, J. Ionic Liquids 
and their Solid-state Analogues as Materials for Energy Generation and Storage. Nat. Rev. Mater. 2016, 1, 15005.

(2) Monteiro, M. J.; Bazito, F. C.; Siqueira, L. J. A.; Ribeiro, M. C. C.; Torresi, R. M. Transport Coefficients, Raman Spectroscopy, and Computer Simulation of Lithium Salt Solutions in an Ionic Liquid. J. Phys. Chem. B 2008, 112, 2102-2109.

(3) Salanne, M. Ionic Liquids for Supercapacitor Applications. Top. Curr. Chem. 2017, $375,63$.

(4) Balducci, A. Ionic Liquids in Lithium-Ion Batteries. Top. Curr. Chem. 2017, 375, 20.

(5) Suo, L.; Hu, Y.-S.; Li, H.; Armand, M.; Chen, L. A New Class of Solvent-in-Salt Electrolyte for High-Energy Rechargeable Metallic Lithium Batteries. Nat. Commun. 2013, 4, 1481.

(6) Yamada, Y.; Yaegashi, M.; Abe, T.; Yamada, A. A Superconcentrated Ether Electrolyte for Fast-Charging Li-ion Batteries. Chem. Commun. 2013, 49, 11194-11196.

(7) Wang, J.; Yamada, Y.; Sodeyama, K.; Chiang, C. H.; Tateyama, Y.; Yamada, A. Superconcentrated Electrolytes for a High-voltage Lithium-ion Battery. Nat. Commun. 2016, 7, 12032.

(8) Yamada, Y.; Usui, K.; Sodeyama, K.; Ko, S.; Tateyama, Y.; Yamada, A. Hydrate-melt Electrolytes for High-Energy-Density Aqueous Batteries. Nat. Energy 2016, 1, 16129.

(9) Suo, L.; Borodin, O.; Gao, T.; Olguin, M.; Ho, J.; Fan, X.; Luo, C.; Wang, C.; Xu, K. "Water-in-Salt" Electrolyte Enables High-voltage Aqueous Lithium-ion Chemistries. Science 2015, 350, 938-943.

(10) Yang, C.; Chen, J.; Qing, T.; Fan, X.; Sun, W.; von Cresce, A.; Ding, M. S.; Borodin, O.; Vatamanu, J.; Schroeder, M. A.; Eidson, N.; Wang, C.; Xu, K. 4.0 V Aqueous Li-Ion Batteries. Joule 2017, 1, 122-132. 
(11) Dubouis, N.; Lemaire, P.; Mirvaux, B.; Salager, E.; Deschamps, M.; Grimaud, A. The Role of the Hydrogen Evolution Reaction in the Solid-Electrolyte Interphase Formation Mechanism for "Water-in-Salt" Electrolytes. Energy Environ. Sci. 2018, 11, 3491-3499.

(12) Yang, C.; Chen, J.; Ji, X.; Pollard, T. P.; Lü, X.; Sun, C.-J.; Hou, S.; Liu, Q.; Liu, C.; Qing, T.; Wang, Y.; Borodin, O.; Ren, Y.; Xu, K.; Wang, C. Aqueous Li-ion Battery Enabled by Halogen Conversion-Intercalation Chemistry in Graphite. Nature 2019, $569,245-253$.

(13) Dubouis, N.; Park, C.; Deschamps, M.; Abdelghani-Idrissi, S.; Kanduc, M.; Colin, A.; Salanne, M.; Dzubiella, J.; Grimaud, A.; Rotenberg, B. Chasing Aqueous Biphasic Systems from Simple Salts by Exploring the LiTFSI/LiCl/H2O Phase Diagram. ACS Cent. Sci. 2019, 5, 640-643.

(14) Borodin, O. et al. Liquid Structure with Nano-Heterogeneity Promotes Cationic Transport in Concentrated Electrolytes. ACS Nano 2017, 11, 10462-10471.

(15) Kashyap, H. K.; Annapuredy, H. V. R.; Raineri, F. O.; Margulis, C. J. How is Charge Transport Different in Ionic Liquids and Electrolyte Solutions? J. Phys. Chem. B 2011, $115,13212-13221$.

(16) Plimpton, S. Fast Parallel Algorithms for Short-Range Molecular Dynamics. J. Comput. Phys. 1995, 117, 1-19.

(17) Berendsen, H. J. C.; Grigera, J. R.; Straatsma, T. P. The Missing Term in Effective Pair Potentials. J. Phys. Chem. 1987, 91, 6269-6271.

(18) Chandrasekhar, J.; Spellmeyer, D. C.; Jorgensen, W. L. Energy Component Analysis for Dilute Aqueous Solutions of Lithium(1+), Sodium(1+), Fluoride(1-), and Chloride(1-) Ions. J. Am. Chem. Soc. 1984, 106, 903-910. 
(19) Wu, D.; Chen, A.; Johnson, Jr., C. S. An Improved Diffusion-Ordered Spectroscopy Experiment Incorporating Bipolar-Gradient Pulses. J. Mag. Res. Series A 1995, 115, 260-264.

(20) Canongia Lopes, J. N.; Deschamps, J.; Pádua, A. A. H. Modeling Ionic Liquids Using a Systematic All-Atom Force Field. J. Phys. Chem. B 2004, 108, 2038-2047.

(21) Yeh, I.-C.; Hummer, G. System-Size Dependence of Diffusion Coefficients and Viscosities from Molecular Dynamics Simulations with Periodic Boundary Conditions. J. Phys. Chem. B 2004, 108, 15873-15879.

(22) Canongia Lopes, J. N.; Pádua, A. A. H. CL\&P: A generic and systematic force field for ionic liquids modeling. Theor. Chem. Acc. 2012, 131, 1129.

(23) Salanne, M. Simulations of Room Temperature Ionic Liquids: from Polarizable to Coarse-Grained Force Fields. Phys. Chem. Chem. Phys. 2015, 17, 14270-14279.

(24) Müller-Plathe, F.; van Gunsteren, W. F. Computer Simulation of a Polymer Electrolyte: Lithium Iodide in Amorphous Poly(Ethylene Oxide). J. Chem. Phys. 1995, 103, 47454756.

(25) Morrow, T. I.; Maginn, E. J. Molecular Dynamics Study of the Ionic Liquid 1-n-Butyl3-methylimidazolium Hexafluorophosphate. J. Phys. Chem. B 2002, 106, 12161-12164.

(26) Bhargava, B. L.; Balasubramanian, S. Refined Potential Model for Atomistic Simulations of Ionic Liquid [Bmim][ $\left.\mathrm{PF}_{6}\right]$. J. Chem. Phys. 2007, 127, 114510.

(27) Dommert, F.; Wendler, K.; Berger, R.; Delle Site, L.; Holm, C. Force Fields for Studying the Structure and Dynamics of Ionic Liquids: A Critical Review of Recent Developments. ChemPhysChem 2012, 13, 1625-1637.

(28) Schröder, C. Comparing Reduced Partial Charge Models with Polarizable Simulations of Ionic Liquids. Phys. Chem. Chem. Phys. 2012, 14, 3089-3102. 
(29) Neumann, J.; Golub, B.; Odebrecht, L.-M.; Ludwig, R.; Paschek, D. Revisiting Imidazolium Based Ionic Liquids: Effect of the Conformation Bias of the $\left[\mathrm{NTf}_{2}\right]$ Anion Studied by Molecular Dynamics Simulations. J. Chem. Phys. 2018, 148, 193828.

(30) Brandt, A.; Pohlmann, S.; Varzi, A.; Balducci, A.; Passerini, S. Ionic Liquids in Supercapacitors. MRS Bulletin 2013, 38, 554-559.

(31) McDaniel, J. G.; Son, C. Y. Ion Correlation and Collective Dynamics in BMIM/BF 4 -Based Organic Electrolytes: From Dilute Solutions to the Ionic Liquid Limit. J. Phys. Chem. B 2018, 122, 7154-7169.

(32) Dong, D.; Bedrov, D. Charge Transport in [Li(tetraglyme)][bis(trifluoromethane) sulfonimide] Solvate Ionic Liquids: Insight from Molecular Dynamics Simulations. J. Phys. Chem. B 2018, 122, 9994-10004.

(33) Wohde, F.; Balabajew, M.; Roling, B. $\mathrm{Li}^{+}$Transference Numbers in Liquid Electrolytes Obtained by Very-Low-Frequency Impedance Spectroscopy at Variable Electrode Distances. J. Electrochem. Soc. 2016, 163, A714-A721.

(34) Yang, Y.; Davies, D. M.; Yin, Y.; Borodin, O.; Lee, J. Z.; Fang, C.; Olguin, M.; Zhang, Y.; Sablina, E. S.; Wang, X.; Rustomji, C. S.; Meng, Y. S. High-Efficiency Lithium-Metal Anode Enabled by Liquefied Gas Electrolytes. Joule 2019, 3, 19862000.

(35) Dong, D.; Sälzer, F.; Roling, B.; Bedrov, D. How Efficient is $\mathrm{Li}^{+}$Ion Transport in Solvate Ionic Liquids under Anion-Blocking Conditions in a Battery? Phys. Chem. Chem. Phys. 2018, 20, 29174-29183.

(36) Urahata, S. M.; Ribeiro, M. C. C. Structure of Ionic Liquids of 1-alkyl-3methylimidazolium Cations: A Systematic Computer Simulation Study. J. Chem. Phys. 2004, 120, 1855-1863. 
(37) Canongia Lopes, J. N. A.; Pádua, A. A. H. Nanostructural Organization in Ionic Liquids. J. Phys. Chem. B 2006, 110, 3330-3335.

(38) Kashyap, H. K.; Hettige, J. J.; Annapuredy, H. V. R.; Margulis, C. J. SAXS AntiPeaks Reveal the Length-Scales of Dual Positive-Negative and Polar-Apolar Ordering in Room-Temperature Ionic Liquids. Chem. Commun. 2012, 48, 5103-5105. 


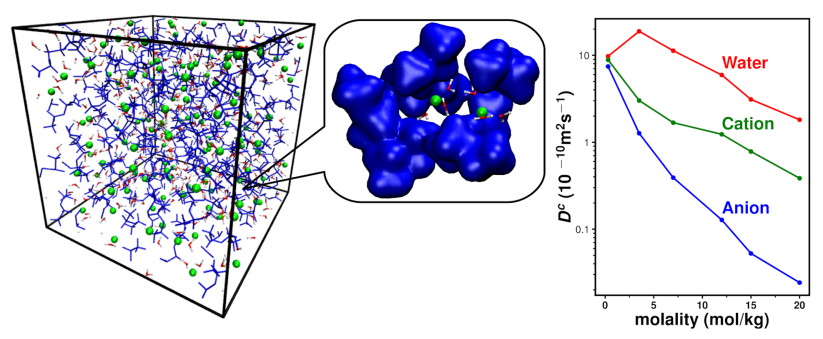

Figure 5: TOC graphic 